Ann. Zootech., I973, 22 (I), I03-rog.

\title{
NYCTHÉMÈRES DE 26 ET 28 HEURES ET DÉPOT DE LA COQUILLE CHEZ LA POULE DOMESTIQUE
}

\author{
L. LACASSAGNE et B. SAUVEUR \\ avec la collaboration technique de C. Bouchot \\ Station de Recherches avicoles. \\ Centre de Recherches de Tours, I. N. R. A., \\ Nouzilly, 37380 Monnaie
}

RÉSUMÉ

Au cours de deux expériences successives effectuées en bâtiments conditionnés en lumière ct tompérature, nous avons étudié les modifications du poids de l'cuf et du poids de coquille par unité de surface (index de coquille) obtenues en soumettant, depuis leur naissance, des poules à des cycles nycthéméraux de 26 heures (I $7,20 \mathrm{~L} / 8,40 \mathrm{O})$ ou de 28 heures $(\mathrm{I} 8,40 \mathrm{~L} / 9,20 \mathrm{O})(60$ animaux par lot).

Dans chaque expérience, le lot témoin soumis à un nycthémère normal de 24 heures, a reçu I6 heures de lumière et 8 heures d'obscurité $(16 \mathrm{~L} / 80$ ) de façon à ce qu'à tout âge la quantité totale de lumière attribuée à chaque lot demeure identique.

Dans ces conditions, il ressort que l'allongement du nycthémère accrồt de façon hautement significative l'index de coquille des lots élevés en cycles de 26 heures et 28 heures. De la même façon, ces nycthémères longs tendent à accroître le poids de l'œuf tout au long de l'année de ponte.

\section{INTRODUCTION}

L'utilisation de cycles nycthéméraux différents de 24 heures dans le but soit d'augmenter l'intensité de ponte de troupeaux de pondeuses soit de sélectionner des animaux aux potentialités supérieures à celles exprimées par les croisements actuels a été tentée à plusieurs reprises (voir à ce sujet 1'article de synthèse de FosTER, I972).

A l'opposé, les faits relatifs à l'incidence de ces nycthémères sur la qualité de la production sont assez rares. Rosales et al. ont publié, en I968, des chiffres relatifs au poids moyen des cuffs obtenus dans des troupeaux soumis à des cycles nycthéméraux de 23,25 et 27 heures, dans lesquels on peut relever que les bonnes pondeuses répondent à un allongement du nycthémère par une augmentation substantielle du poids de l'œuf. Ce phénomène a été confirmé depuis par Fox et al. en I97I. 


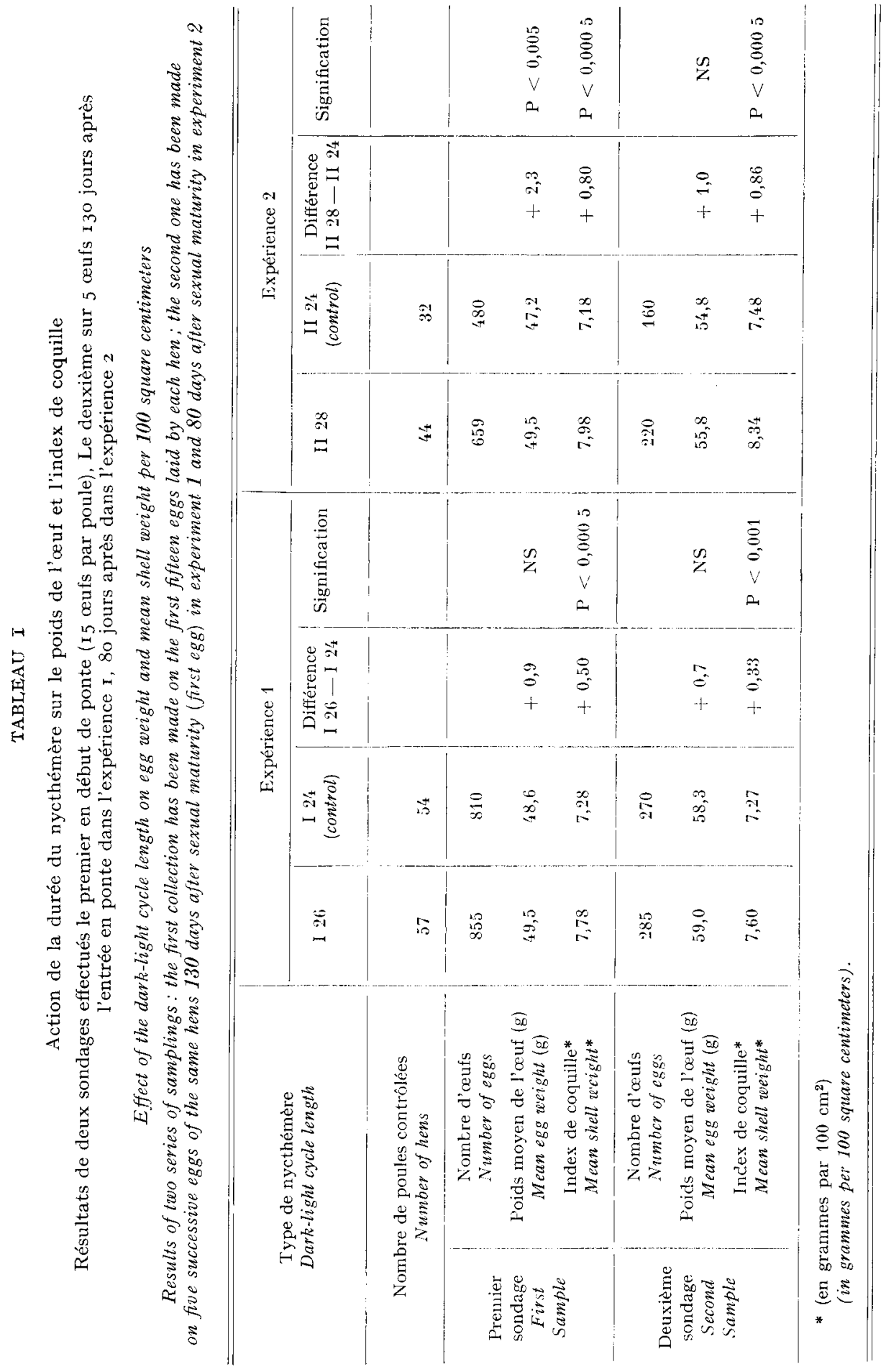


En ce qui concerne la coquille, VAN AlbadA (I958) a mentionné une augmentation de son épaisseur chez des poules soumises à des nycthémères de 26 heures. Aucun autre travail n'ayant été, semble-t-il, publié depuis sur ce point précis, nous rapportons ci-après les modifications qualitatives de production obtenues dans des troupeaux de pondeuses élevés en nycthémères de 26 et 28 heures.

\section{MATÉRIEL, ET MÉTHODES}

Au cours de deux expériences successives, nous avons élevé au total $24^{\circ}$ poules Leghorn blanches de type Heisdorf-Nelson réparties en 4 lots de 60 animaux.

La première expérience effectuée du 9-I-67 au 4-2-68 comportait un lot témoin (I 24) en nycthémère de 24 heures : I 6 heures de lumières (L) et 8 heures d'obscurité (O), et un lot expérimental ( $\mathrm{I} 26)$ en cycle nycthéméral de 26 heures $(17,20 \mathrm{~L} / 8,40 \mathrm{O})$. La deuxième expérience (du 2-7-68 au 27-3-69) comportait un lot témoin similaire (II 24) et un lot expérimental (II 28) en cycle nycthéméral de 28 heures ( $18,40 \mathrm{~L} / 9,20 \mathrm{O}$ ). Dans tous les lots le rapport de la durée de la période claire à la durée de la période obscure était égal à 2 de façon qu'à tout âge la quantité totale de lumière reçue par chaque animal depuis sa naissance soit identique.

Les poulettes, tout d'abord élevées au sol dans des bâtiments conditionnés en lumière, ont été transférées 4 semaines au moins avant leur maturité sexuelle, dans des locaux insonorisés, à lumière contrôlée dont la température et l'hygrométrie constantes étaient régulées automatiquement. Durant les périodes de croissance et de ponte l'aliment a été donné ad libitum.

Au cours de la deuxième expérience les animaux du lot 28 heures ont subi, durant leur croissance, une attaque de coccidiose sévère suivie d'une forte mortalité par maladie de Marek à l'âge adulte ( 2 p. Ioo par semaine).

Dans chaque expérience, l'index de coquille a été évalué au cours de deux sondages dont le premier concernait les I 5 premiers cufs de chaque poule. Le deuxième sondage portant sur cinq œufs consécutifs de ces mêmes poules fut effectué i zo jours après la date correspondant à Ie maturité sexuelle moyenne du troupeau pour I 24 et I 26 et 80 jours après cette même date pour II 24 et II 28. Seuls ont été retenus les animaux dont nous possédions des résultats complets pour les deux sondages.

Le poids en grammes de $100 \mathrm{~cm}^{2}$ de coquille (index de coquille) a été calculé selon Hurwitz et Griminger (I962), la surface de l'œuf étant évaluée à l'aide de la formule S = KP2/3 proposée par Mueller et Scott (I940) où $K$ prend les valeurs 4,67-4,68-4,69 lorsque le poids varie de 55 à $75 \mathrm{~g}$ (Bonnet et Mongin, 1965). En l'absence de coefficients connus pour les poids inférieurs à $55 \mathrm{~g}$, nous avons affecté arbitrairement au coefficient $\mathrm{K}$ les valeurs 4,66 et 4,65 pour les oufs compris entre 55 et $35 \mathrm{~g}$. Les résultats obtenus ont été comparés par inalyse de variance dans laquelle étaient contrôlées les variabilités entre œufs d'une même poule, entre poules d'un même lot et entre lots.

\section{RÉSULTATS}

Les résultats relatifs au poids de l'œuf et à l'index de coquille sont regroupés au tableau ci-contre.

\section{Poids de l'ouf}

Qu'il s'agisse du premier ou du deuxième sondage, nous constatons qu'en nycthémère long (I 26 et II 28 ) les poules ont tendance à pondre des œufs de poids supérieur à celui des œufs des poules témoins. Par rapport à celui-ci, l'augmentation est de 0,9 et $0,7 \mathrm{~g}$ pour le premier et le deuxième sondage respectivement dans le lot I 26 et de 2,3 $\mathrm{g}$ de $\mathrm{I}, \mathrm{O} \mathrm{g}$ pour les sondages correspondant du lot II 28 . Dans les conditions où nous nous sommes placés pour cette analyse, seule la différence de 2,3 $\mathrm{g}$ entre II 28 et II 24 en début de ponte est significative. Ce point sera discuté plus loin. 


\section{Index de coquille}

Dans les deux cas étudiés, l'allongement de la durée du nycthémère augmente de façon très nette l'index de coquille. Dans la première expérience, les œufs pondus en nycthémère de 26 heures présentent, par rapport aux œufs obtenus en 24 heures, un index moyen supérieur de 0,50 dans le premier sondage et de 0,33 dans le second. L'écart observé entre les index moyens des œufs du lot II 28 et ceux du lot II 24 est encore plus important. Il est de 0,80 en début de ponte et de 0,86 ensuite. Rapporté à un ouf pesant 48,57 $\mathrm{g}$ (moyenne des deux lots), dont la surface moyenne est de $62,06 \mathrm{~cm}^{2}$, cet écart d'index reflète au moment du premier sondage une différence de $0,50 \mathrm{~g}$ du poids de coquille en faveur du lot II 28 . Sa valeur atteint $0,58 \mathrm{~g}$ I 30 jours après (poids moyen $55,42 \mathrm{~g}$, surface moyenne $67,87 \mathrm{~cm}^{2}$ ). En ce qui concerne la première expérience et sur les mêmes bases, l'écart d'index observé en faveur du lot I 26 représente $0,3 \mathrm{I} \mathrm{g}$ de coquille au premier sondage (cuf de poids moyen 49, I $\mathrm{g}$, de surface $62,49 \mathrm{~cm}^{2}$ ) et $0,24 \mathrm{~g}$ au deuxième (œuf de $58,6 \mathrm{~g}$, surface $70,45 \mathrm{~cm}^{2}$ ).

Toutes ces différences sont en valeur absolue fort importantes et hautement significatives.

\section{DISCUSSION}

Bien que la différence de 2,3 g entre le poids des œufs pondus par les poules des lots II 28 et II 24 en début de ponte soit seule significative, nous pensons que l'augmentation de poids relevée dans les trois autres sondages est bien réelle ; le défaut de signification statistique peut être attribué pour le $2^{\mathrm{e}}$ contrôle à la faiblesse de l'échantillonnage et pour le $\mathrm{I}^{\mathrm{cr}}$ contrôle à la variabilité du poids des cufs pondus par une même poule au début de la ponte (premiers cufs de poids souvent relativement faible et présence de I à 2 p. Ioo d'œufs à double jaune). La répartition des œufs par classe de poids tout au long de chaque expérience nous confirme dans cette idée. Ces résultats rejoignent ceux de Rosales et al. (I968), Fox ct al. (I97I) et de MorRis et Fox (I97I). Comme ces derniers auteurs, nous constatons en II 28 que l'accroissement du poids de l'cuf par rapport aux valeurs du lot témoin est inportant en début de ponte et s'atténue par la suite.

L'allongement du cycle nycthéméral induit de façon certaine un accroissement de la quantité de coquille déposée sur l'œuf. Nos résultats confirment donc ceux deVAN Al,BADA (I958) pour les nycthémères de 26 heures et montrent qu'un phénomène identique mais amplifié se retrouve en nycthémère de 28 heures.

En première analyse on peut se demander si l'augmentation de l'épaisseur de coquille ainsi obtenue provient du retard de maturité sexuelle présenté par les animaux soumis aux cycles longs. Les programmes qui entraînent une entrée en ponte tardive permettent en effet d'obtenir une augmentation d'index de coquille en début de ponte (LaCASSAGne et Mongin, I965, en nycthémère normal; Marks et LuCAS, I967, en nycthémère de I8 heures). Dans nos résultats de I965, l'accroissement de cet index n'était significatif que pour un retard d'entrée en ponte de 24 jours, un retard de 8 jours induisant une augmentation d'index non significative. Sans que l'on puisse écarter complètement son éventualité, un tel mécanisme ne semble pas 
être mis en jeu dans l'expérience présente du fait du faible retard de maturité sexuelle observé en nycthémères longs : retard de ro jours du lot II 28 par rapport au lot II 24 et de 8 jours du lot I 26 par rapport au lot I 24 . Cela d'autant plus que TAyLor et Morris (I964) n'ont pu mettre en évidence, à 2 I9 jours d'âge, une différence d'épaisseur de coquille dans la ponte de deux troupeaux éclairés de manière à obtenir des maturités sexuelles fort différentes (I 45 jours et I 78 jours) malgré une action évidente du traitement sur le métabolisme calcique.

L'origine de l'augmentation de l'index de coquille peut également être recherchée dans un abaissement de la production d'œufs (SIEGEL et BEANE, I963). Cette explication ne peut être écartée pour le lot II 28 dont l'intensité de ponte s'est avérée bien inférieure à celle du lot témoin durant toute la durée de l'expérience : intensité de 7 I,6 et 84,6 pour les lots II 28 et II 24 respectivement à l'époque du deuxième sondage. Nous n'avons pas retenu l'intensité de ponte observée pendant la période de production des I5 premiers cufs, la jugeant dépourvue de toute signification.

A l'opposé, ce mécanisme ne peut être invoqué pour le lot I 26 dont l'intensité de ponte est demeurée à tout moment identique à celle du lot témoin. L'intensité de ponte était de $8 \mathrm{r}, 4$ et 84,2 pour I 26 et I 24 respectivement durant les quatre semaines recouvrant la période du deuxième sondage.

Nous pensons plutôt que l'augmentation d'index observée en nycthémères longs résulte de la prolongation du séjour de l'œuf dans l'utérus au-delà du temps habituellement observé pour des intensités de ponte analogues. Cette hypothèse s'appuie sur la convergence de faits présentée ci-après.

Le temps qui s'écoule entre l'oviposition de deux œufs consécutifs s'accroît et devient plus homogène lorsque la période du nycthémère augmente. I,e phénomène est très net dès que la période atteint une valeur de 26 heures (VAN ALBADA, I958, RosAlEs et al., I968, LACASSAGNE, I970). Cet allongement du temps qui sépare deux ovipositions consécutives ne semble attribuable, du moins pour l'essentiel, ni au retard de 1'ovulation d'un œuf par rapport à l'oviposition de l'œuf précédent, ni à une grande réduction de la vitesse de transit du jaune dans le magnum. En effet, en nycthémère de 26 heures le jaune ne parvient dans la partie terminale du magnum que I 2 minutes environ plus tard qu'en jour normal alors que les intervalles entre ovipositions sont en moyenne plus longs de 67 minutes en nycthémère de 26 heures qu'en 24 heures (Lacassagne, I970). L'essentiel de la différence de temps qui sépare deux ovipositions dans l'un et 1'autre cas provient donc du séjour de l'œuf dans l'isthme et l'utérus et, plus vraisemblablement, du séjour dans l'utérus étant donné la rapidité de transit de l'œuf à travers l'isthme. Nous rejoignons là les conclusions auxquelles WARREN et SCOTT (I935 $a$ et $b$ ) étaient parvenus en étudiant la chronologie de la formation de l'œuf chez des poules présentant des intensités de ponte individuelles différentes.

D'autre part, statistiquement, les cufs qui constituent une même série présentent, en fonction de leur rang, des caractéristiques particulières quant à l'épaisseur de la coquille et au temps qui sépare l'oviposition de l'un d'entre eux de celle de l'œuf qui précède. BERG a montré en I945 qu'il existait une corrélation positive entre ces deux paramètres et que la différence d'épaisseur de coquille observée entre les œufs d'une même série pouvait découler de la différence du temps de séjour de ces œufs dans l'oviducte. L'effet d'une diminution de l'intensité de ponte peut généralement s'interpréter de façon identique car, lorsque la longueur de la série diminue, le temps 
de séjour de l'œuf dans l'oviducte s'accroît. Si cette hypothèse se confirme, l'attention se trouvera de nouveau attirée sur la nature des mécanismes mis en jeu dans l'arrêt de la sécrétion de carbonate de calcium au niveau de l'utérus dans les heures qui précèdent l'oviposition.

Dans un autre ordre d'idées, il faut également penser que la simultanéité de l'absorption intestinale du calcium et de la formation de la coquille peut retentir sur la solidité de cette dernière (Scott et Mullenhoff, I970).

Or, dans l'expérience présente, l'heure moyenne d'oviposition ne se situe pas de façon identique par rapport à la période diurne dans les deux nycthémères. En cycle nycthéméral de 28 heures, l'oviposition a lieu en moyenne 4 heures avant l'allumage de la lumière alors qu'en 24 heures le mode de l'histogramme de répartition des ovipositions en fonction de l'heure se situe 6 heures après l'allumage.

Il est probable que ce décalage entre les heures d'alimentation et la formation de la coquille soit aussi intervenu en faveur d'une coquille plus épaisse dans le lot 28 heures en supposant que le comportement alimentaire des animaux soit demeuré le même dans les deux nycthémères.

Recu pour publication en novembre 1972.

\title{
SUMMARY
}

\author{
I,IGHT-DARK CYCLES OF 26 AND 28 HOURS \\ AND EGG SHELI, FORMATION IN THE DOMESTIC HEN
}

In two experiments under controlled conditions of light and temperature, we have studied the effect of two light-dark cycles on egg weight and egg shell quality (shell weight per Ioo $\mathrm{cm}^{2}$ ). The length of the first cycle was 26 hours with 17.20 hours of light (L) and 8.40 hours of darkness (O) and that of the second one was 28 hours with 18.40 hours and 9.20 hours of light and darkness respectively.

In each experiment, the control group was subjected to a 24 hours cycle (I6 hours light and 8 hours darkness). In each of the four groups of 60 animals the ratio between light and darkness was constant in order to give the same amount of light at any given age.

Under these conditions, an increase of the length of light-dark cycle (significantly) improves the shell quality regardless of the age of the laying hens. The egg weight also seems to be improved.

\section{RÉFÉRENCES BIBLIOGRAPHIQUES}

BERG L. R., I945. The relationship of clutch position and time interval between eggs to eggshell quality. Poult. Sci., 24, 555-563.

Bonnet Y., Monain P., i965. Mesure de la surface de l'ceuf. Ann. Zootech., 14, 3II-317.

Foster W. H., I972. Production and selection under light dark cycles of abnormal lengths. In FREEman B. H., Lake P. E., Egg formation and production, 161-183. British Poult. Sci., Ltd, Edinburgh.

Fox S., Morris T. R., Jennivgs R. C., r97I. The use of non 24 hour cycles to manipulate egg weight in pullets. World's Poult. Sci. J., 27, 159. (Abstr.).

Hurwitz S., Griminger P., Ig62. Eggg production and shell quality in temperature and light controlled versus incontrolled environmement. Poull. Sci., 4.1, 499-508.

Lacassagne L., I970. Analyse du déterminisme de la formation des séries chez la poule à l'aide de cycles nycthéméraux de 21 et 26 heures. Ann. Biol. anim. Bioch. Biophys., 10, 59-7 I.

Lacassagne L., Mongin P., i965. Maturité sexuelle et qualité de la coquille de l'œuf. Ann. Zootech., 14, 327-334. 
MARKS H. L., Lucas L. M., I967. The influence of early photoperiod lengths on birds maintained under "short days". Poult. Sci., 46, I589-I59o.

Morris T. R., Fox S., I97I. Ahemeral light-dark cycles and egg weight in the fowl. Poult. Testing., $1,7 \mathrm{I}-73$.

Mufller C. D., Scott H. M., I94o. The porosity of egg shell on relation to hatchability. Poult. Sci., 19, I63-I66.

Rosales A. A., Biellier H. V., Stephenson A. B., i968. Effect of light-cycles on ovipositions and egg production. Poult. Sci., 47, 586-591.

Scott M. L., Mullenhoff P. A., r97o. Dietary oyster shell and eggshell quality. Proc. Cornell Nutr. Conf. Fd. Manuf., 24-28.

Siegel H. S., Beane W. L., I964. Photoperiod length and shell thickness in laying fowl. Poult. Sci., 43, I36r-I362 (Abstr.).

TAYLOR T. G., Morris T. R., I964. The effect of early and late maturity on the skeletons of pullets. World's Poult Sci. J., 20, 294-297.

VAN AlBada M., I958. Seasonal and lighting influences on the laying rythm of the fowl. Intern. J. Bioclimatol. Biomet., 2 Part III, Section A, I-8.

Warren D. C., Scott H. M., r935 a. The time factor in egg formation. Poult. Sci., 14, r95-207.

Warren D. C., ScotT I. M., I 935 b. Physiological factors influencing the rate of egg formation in the domestic hen. J. Agric. Res., 51, 565-572. 